\title{
Erratum to: Domain Size Distribution in Segregating Binary Superfluids
}

\section{Hiromitsu Takeuchi ${ }^{1}$}

\section{Erratum to: J Low Temp Phys (2016) 183:169-174 DOI 10.1007/s10909-016-1543-7}

In the original article, there was an error in Eq. 5. Following is the corrected equation:

$$
\rho(S, l) l(t)^{4}=c_{S} \tilde{S}^{-\tau} \equiv \tilde{\rho}(\tilde{S})
$$

In addition, there were errors in the vertical axes of Figs. 1 and 2. Following are the corrected figures.

The online version of the original article can be found under doi:10.1007/s10909-016-1543-7.

$凶$ Hiromitsu Takeuchi hirotake@sci.osaka-cu.ac.jp

1 Department of Physics, Osaka City University, 3-3-138 Sugimoto, Sumiyoshi-ku, Osaka 558-8585, Japan 
Fig. 1 Domain size distribution $\rho_{0}(S)$ in the initial pattern with $l=l_{0}$. A broken line represents the power law with the

approximate value $\tau=2$ of the Fisher exponent. The

distribution $\rho_{0}(S)$ obeys the power law in the scaling regime $l_{0}^{2} \ll S \ll L^{D_{S}}$ with the fractal dimension $D_{S}=2-\beta / \nu$ with the critical exponents $\beta=5 / 36$ and $v=4 / 3$ of two-dimensional percolation theory

Fig. 2 Dynamic scaling plot of the domain size distribution $\rho(S, l)$ for $l(t) / l_{0}=$ $0.8,1.0,1.4,2.0,2.8,3.9$, and 5.6 with the effective system sizes $\tilde{L}=L / l(t)=$ 81.5, 65.2, 46.6, 32.6, 23.3, 16.7, and 11.6 , respectively. The broken line represents the universal function $\tilde{\rho}(\tilde{S})$ with $c_{S}=0.1$ and $\tau=2$. The positions of $\tilde{S}=\tilde{L}^{D_{S}}$ for different values from $l(t) / l_{0}=0.8$ to $l(t) / l_{0}=5.6$ are represented by thick arrows from right to left (Color figure online)
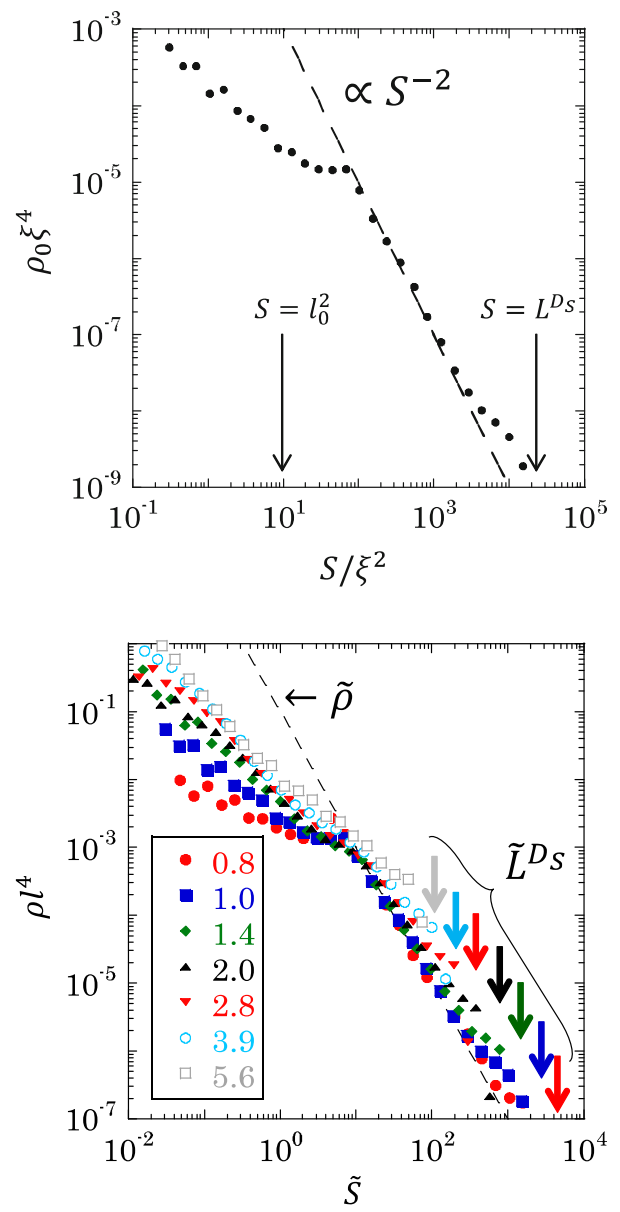